\title{
Short communication: Ability of dogs to detect cows in estrus from sniffing saliva samples
}

\author{
C. Fischer-Tenhagen, ${ }^{*}$ B.-A. Tenhagen, $\dagger$ and W. Heuwieser ${ }^{* 1}$ \\ ${ }^{*}$ Clinic for Animal Reproduction, Freie Universität Berlin, 14163 Berlin, Germany \\ †Bundesinstitut für Risikobewertung, 12277 Berlin, Germany
}

\begin{abstract}
Efficient estrus detection in high-producing dairy cows is a permanent challenge for successful reproductive performance. In former studies, dogs have been trained to identify estrus-specific odor in vaginal fluid, milk, urine, and blood samples under laboratory conditions with an accuracy of more than $80 \%$. For on-farm utilization of estrus-detection dogs it would be beneficial in terms of hygiene and safety if dogs could identify cows from the feed alley. The objective of this proof of concept study was to test if dogs can be trained to detect estrus-specific scent in saliva of cows. Saliva samples were collected from cows in estrus and diestrus. Thirteen dogs of various breeds and both sexes were trained in this study. Five dogs had no experience in scent detection, whereas 8 dogs had been formerly trained for detection of narcotics or cancer. In the training and test situation, dogs had to detect 1 positive out of 4 samples. Dog training was based on positive reinforcement and dogs were rewarded with a clicker and food for indicating saliva samples of cows in estrus. A false indication was ignored and documented in the test situation. Dogs with and without prior training were trained for 1 and $5 \mathrm{~d}$, respectively. For determining the accuracy of detection, the position of the positive sample was unknown to the dog handler, to avoid hidden cues to the dog. The overall percentage of correct positive indications was $57.6 \%$ (175/304), with a range from $40(1 \mathrm{dog})$ to $75 \%$ (3 dogs). To our knowledge, this is the first indication that dogs are able to detect estrus-specific scent in saliva of cows.
\end{abstract}

Key words: estrus detection, saliva, detection dog

\section{Short Communication}

Efficient estrus detection is a permanent challenge for successful reproductive performance in dairy cattle.

Received May 2, 2012.

Accepted November 1, 2012.

${ }^{1}$ Corresponding author: w.heuwieser@fu-berlin.de
Traditional estrus detection via visual observation is time consuming and ineffective (Peralta et al., 2005). Time spent for observation and experience of the observer are crucial for achieving acceptable estrusdetection rates. Behavioral changes, such as mounting, standing to be mounted, and increase of activity are indicators of cows in estrus. Standing to be mounted is most predictive for cows in estrus, but only $60 \%$ of cows in estrus express this behavior (Roelofs et al., 2005). Numerous estrus-detection aids, such as activity meters and mounting detectors, have been developed. Reported estrus detection accuracies of these devices vary from under $49.3 \%$ (Peralta et al., 2005) to over 86.8\% (At-Taras and Spahr, 2001).

It is well known that dogs have the ability to smell cows in estrus (Kiddy et al., 1978; Jezierski, 1992). Specifically trained dogs have been used to distinguish between swabs with vaginal fluid, urine, blood, and milk (Hawk et al., 1984; Kiddy et al., 1984) from cows in estrus and diestrus. In a recent study, we demonstrated that dogs can reach an accuracy of $80.3 \%$ after only 1 wk of training using a special training platform (Fischer-Tenhagen et al., 2011). All of these studies were performed with urine or vaginal mucus samples collected from dairy cows and investigated under laboratory conditions (i.e., outside the barn). To identify such estrus-specific odors directly at cowside would require using a dog from behind the cows. Obviously, the disadvantages of such an approach include risk of injuries, hygienic problems, and a more stressful experience for the cows. Identifying cows in estrus from the feed alley with the cows fixed in the head locks would be advantageous. Sankar and Archunan (2008) demonstrated estrus-specific volatile substances in the saliva of cows using a bioassay with rats. It has not yet been studied if dogs are able to identify estrus-specific odor in saliva samples of cows. Therefore, the objective of this study was to demonstrate that dogs can identify cows in estrus from sniffing saliva samples. Specifically, aims were (1) to show that dogs can identify estrusspecific odor in the saliva of cows and (2) to demonstrate that estrus-detection accuracy of this approach is at least $80 \%$. 
Thirteen dogs of various breeds were enrolled in this experiment. All dogs were privately owned and had basic experience with scent detection. Seven dogs were trained for personal reasons (pet dogs), whereas 6 dogs were part of a scent-detection dog team owned by volunteers (professional dogs). Selection of dogs was by convenience.

Saliva samples were collected on 2 commercial dairy farms in Brandenburg, Germany, milking 200 and 400 cows, respectively. Cows were housed in a freestall barn on deep-bedded straw. For estrus samples, cows were selected when displaying signs of estrus, such as standing firm when mounted by another cow and showing clear and viscous vaginal mucous. After collecting the samples, cows were inseminated within $2 \mathrm{~h}$. Between 35 to $41 \mathrm{~d}$ postinsemination, pregnancy was checked by transrectal palpation. For training purposes, samples were used only if the cow conceived from the insemination to ensure correct scent conditioning. Samples serving as negative controls were drawn from cows with a prominent corpus luteum, diagnosed by ultrasound (BCF easy scan; BCF Technology Ltd., Livingstone, UK) on at least 1 ovary but without any prominent follicle and without any signs of estrus.

Saliva (5 to $10 \mathrm{~mL}$ ) was collected in a disposable sterile plastic container $(100 \mathrm{~mL})$ by opening the cow's mouth, pulling out the tongue, and catching saliva dripping into the container. Disposable vinyl gloves were used and changed for every cow. In the laboratory the samples were centrifuged for $3 \mathrm{~min}$ at 2,100 $\times g$ and aliquots of $1 \mathrm{~mL}$ were filled in disposable sterile containers and frozen at $-25^{\circ} \mathrm{C}$ until use. To avoid cross-contamination of samples, disposable vinyl gloves were used throughout and changed each time a new sample was handled.

Training took place in an indoor laboratory (Hundcampus training center, Hällefors, Sweden), using a special training device as previously described (FischerTenhagen et al., 2011). In brief, scent training was adapted from training dogs for special scent tasks (i.e., mine detection and drugs or narcotic search) and comprised a total of 3 modules (i.e., adaptation to the laboratory and training methods, conditioning to estrusspecific cow scents, and actual discrimination training). The training device included a platform $(80 \times 200 \mathrm{~cm})$ covered with a rubber mat for the dogs to stand on and a movable sledge with 7 jars with perforated lids, each located $20 \mathrm{~cm}$ apart. The sledge was covered with a metal plate with only 4 holes for the dog to sniff through. The sledge could be moved to either side by the dog trainer, allowing the presentation of a variable combination of jars to the dog in rapid succession. Thus, the dogs learned to check all 4 holes to point out the positive sample. Standing still and pointing with the nose at the right hole, the dog was rewarded with a clicking noise and a food treat. Dogs were conditioned to the estrus scent using samples collected from cows during the estrus cycle and diagnosed pregnant after AI. Then, the actual discrimination training started when negative samples (cotton swabs soaked in saliva from a cow in diestrus) were introduced.

After the training phase, each dog's accuracy for detection of estrus in saliva was determined in a test laboratory using a similar platform. The sledge, however, carried 16 jars and was operated by an assistant. To avoid bias, the position of the positive sample was blinded to the dog handler and assistant. In each test trial the dog had to identify 1 positive sample out of 4 . This was replicated 4 times. In every replica, a fresh set of samples was used.

Samples were randomly positioned on the sledge by rolling dice. To avoid matrix effects, the samples used in the tests were from different set of individual cows and had not been used previously in the training trials.

An indication was considered as correct positive when the dog had identified the target jar (i.e., saliva sample collected from a cow in estrus) correctly and considered as false positive when the dog had identified a jar loaded with a negative control (i.e., saliva sample collected from a cow in diestrus). Likewise, false negatives were recorded for not identifying the target jar and correct negatives for not indicating the negative samples. It was possible that a dog made no indication in a replicate.

All statistical analyses were carried out using SPSS software (version 19; SPSS Inc., Munich, Germany). The association of the dog and the sample on the test result was studied using logistic regression, with the correct identification of the estrus sample as positive outcome (1) and failure to identify the sample as the alternative outcome (0). The analysis included test trial number and dog as categorical predictors. This analysis included the pet dogs only, as all pet dogs were tested on the same samples, whereas the professional dogs were tested on another day and, thus, had different samples. The effect of dog type (pet vs. professional dog) was also tested using logistic regression with the same outcome. The analysis included the type of dog and the individual dog as categorical predictors.

One of the 7 pet dogs was withdrawn due to nervousness on the test platform. The remaining 4 and 2 pet dogs completed 8 (32 positive out of 128 samples) and 7 (28 out of 112) test trials conducted on 2 consecutive days, respectively. All professional dogs were tested in 5 test trials (20 out of 80) conducted on another 2 consecutive days. One test trial included 4 replicates in which 1 positive out of 4 samples had to be identified by the dogs. 


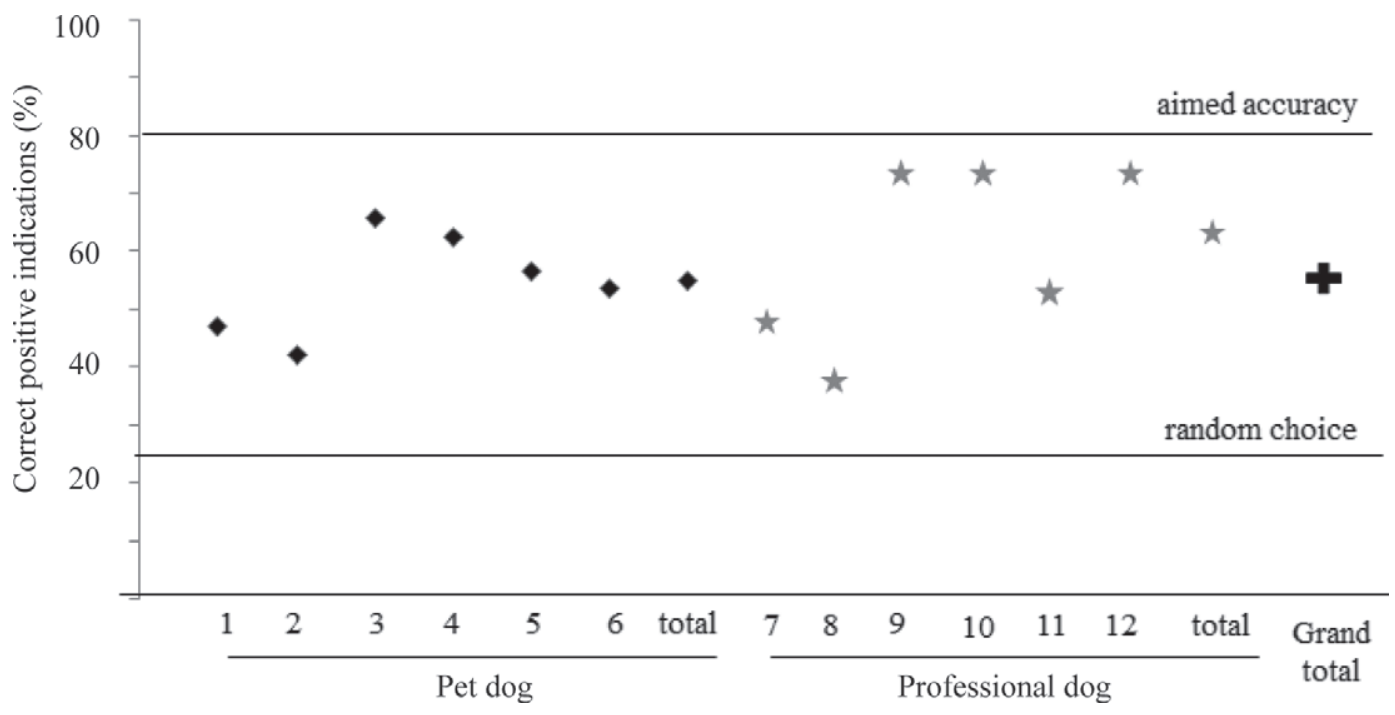

Figure 1. Accuracy of trained dogs for identifying estrus samples.

Results did not differ significantly between test trials $(P=0.07)$. There was no indication of an effect of dog type on the outcome $(P=0.66)$. However, the professional dogs were tested on different samples on a different day. Therefore, this result needs to be confirmed in further studies. Although differences existed between individual dogs, no overall dog effect was observed on the outcome $(P=0.16)$.

The overall accuracy for identifying estrus samples was $57.6 \%(175 / 304)$, with a range from $40.0 \%(8 / 20)$ to $75.0 \%$ (15/20; Table 1 and Figure 1$)$. These percentages of correctly identified samples were higher than

Table 1. Number of correct positive indications of dogs detecting estrus-specific odor in saliva samples of cows

\begin{tabular}{lccc}
\hline & & \multicolumn{2}{c}{ Correct positive indications } \\
\cline { 3 - 4 } Dog & $\begin{array}{c}\text { Number of } \\
\text { replicates }\end{array}$ & Number & $\%$ \\
\hline Pet dogs & & & \\
1 & 32 & 15 & 46.9 \\
2 & 28 & 12 & 42.2 \\
3 & 32 & 21 & 65.6 \\
4 & 32 & 20 & 62.5 \\
5 & 32 & 18 & 56.6 \\
6 & 28 & 15 & 53.6 \\
Total & 184 & 101 & 54.8 \\
Professional dogs & & & \\
7 & 20 & 10 & 50.0 \\
8 & 20 & 8 & 40.0 \\
9 & 20 & 15 & 75.0 \\
10 & 20 & 15 & 75.0 \\
11 & 20 & 11 & 55.0 \\
12 & 20 & 15 & 75.0 \\
Total & 120 & 74 & 61.6 \\
Grand total & 304 & 175 & 57.6 \\
\hline
\end{tabular}

${ }^{1}$ Each replicate consisted of 1 positive and 3 negative samples. would be expected if dogs had picked the samples randomly (25\%).

These results demonstrate that dogs can be trained to detect estrus-specific odors in saliva samples of dairy cows, as addressed in hypothesis 1. Obviously, the overall accuracy was not satisfactory and the second hypothesis that dogs achieve an estrus detection accuracy of $80 \%$ has to be rejected.

In previous studies, dogs achieved estrus-detection rates of 80 to $97 \%$ using vaginal fluid, urine, blood serum, and milk also collected from cows standing to be mounted (Kiddy et al., 1984; Jezierski, 1992; FischerTenhagen et al., 2011). We suppose that estrus-specific substances are more difficult to identify in saliva than in vaginal fluid. This is in agreement with Sankar and Archunan (2005), who found that mice could discriminate estrus-specific odor significantly better in vaginal fluid than in saliva. This could also be the reason for more variability between dogs.

For this study, we followed the same training schedule as for vaginal fluid (Fischer-Tenhagen et al., 2011) and trained the dogs for only $1 \mathrm{wk}$. It can be speculated that a more intensive training period would improve the accuracy, as has been suggested previously (Williams and Johnston, 2002). The accuracy of pet and professional dogs was similar. However, 3 of the professional dogs achieved an accuracy of $75.0 \%(\mathrm{n}=20)$, whereas the highest accuracy in the group of the pet dogs was $65.6 \%(\mathrm{n}=32)$. To the best of our knowledge, this is the first report demonstrating that dogs can identify estrus-specific odor in saliva of cows. However, dogs did not achieve an estrus detection rate of $80 \%$, as they did with vaginal fluid samples. Further research is warranted to evaluate if more intensive training can 
increase detection rates. Finally, the efficiency of dogs on farm (i.e., cowside from the feed alley) has to be tested because sampling saliva for the detection of cows in estrus is impractical for commercial dairy farms.

\section{ACKNOWLEDGMENTS}

We thank Lennart Wetterholm (Hundcampus, Hällefors, Sweden) for his superb cooperation and the Förderverein für Biotechnologie e.V. (Bonn, Germany) for their financial support.

\section{REFERENCES}

At-Taras, E. E., and S. L. Spahr. 2001. Detection and characterization of estrus in dairy cattle with an electronic heatmount detector and an electronic activity tag. J. Dairy Sci. 84:792-798.

Fischer-Tenhagen, C., L. Wetterholm, B.-A. Tenhagen, and W. Heuwieser. 2011. Training dogs on a scent platform for oestrus detection in cows. Appl. Anim. Behav. Sci. 131:63-70.
Hawk, H. W., H. H. Conley, and C. A. Kiddy. 1984. Estrus-related odors in milk detected by trained dogs. J. Dairy Sci. 67:392-397.

Jezierski, T. 1992. Effectiveness of estrus detection in cows by a trained dog. Anim. Sci. Papers Reports 10:57-65.

Kiddy, C. A., D. S. Mitchell, D. J. Bolt, and H. W. Hawk. 1978. Detection of estrus-related odors in cows by trained dogs. Biol. Reprod. 19:389-395.

Kiddy, C. A., D. S. Mitchell, and H. W. Hawk. 1984. Estrus-related odors in body fluids of dairy cows. J. Dairy Sci. 67:388-391.

Peralta, O. A., R. E. Pearson, and R. L. Nebel. 2005. Comparison of three estrus detection systems during summer in a large commercial dairy herd. Anim. Reprod. Sci. 87:59-72.

Roelofs, J. B., F. J. C. M. van Eerdenburg, N. M. Soede, and B. Kemp. 2005. Various behavioral signs of estrous and their relationship with time of ovulation in dairy cattle. Theriogenology 63:1366-1377.

Sankar, R., and G. Archunan. 2005. Discrimination of bovine estrusrelated odors by mice. J. Ethol. 23:147-151.

Sankar, R., and G. Archunan. 2007. Identification of putative pheromones in bovine (Bos taurus) faeces in relation to estrus detection. Anim. Reprod. Sci. 103:149-153.

Williams, M., and J. M. Johnston. 2002. Training and maintaining the performance of dogs (Canis familiaris) on an increasing number of odor discriminations in a controlled setting. Appl. Anim. Behav. Sci. 78:55-65. 